\title{
Clinical patterns and risk factors of vulvo-vaginal candidiasis among women of reproductive age attending a tertiary hospital in central India
}

\author{
Rafat Siddiqui* \\ Department of Microbiology, Chirayu Medical college and hospital, Bhopal, India
}

Received 20 November 2019/Accepted 18 December 2019

\begin{abstract}
Vulvo-vaginal Candidiasis (VVC) is the most common fungal infection in women of reproductive age. Data related to distribution and risk factors are very limited in India. This study was designed to observe the prevalence of $\mathrm{VVC}$ among women of reproductive age group, find the species of Candida causing such infection and find the risk factors associated with VVC. All female patients in the childbearing age group, fulfilling the clinical criteria of vaginitis, reported between January 2016 and June 2017 were included in this study. Standard procedures were followed to collect vaginal swabs. Culture and microscopic examinations were done to isolate Candida albicans and non-albicans Candida (NAC) from the specimens. Descriptive and analytic statistics was used to illustrate the basic and disease characteristics of the study participants. The odds-ratio (OR) associated with each potential risk factor at $95 \%$ confidence interval $(\mathrm{CI})$ were calculated. All results were considered significant at $P<0.05$. Out of total 168 subjects, $32.7 \%$ showed pure growth of $C$ andida species and NAC species were found to be predominant $(65.4 \%)$ followed by $C$. albicans $(34.6 \%)$. Maximum Candida positivity was found in age group 21-30 years $(60 \%)$ compared to other age groups $(P<0.05)$. Candida positivity was found to be higher among pregnant $(45.0 \%)$ than non-pregnant $(28.9 \%)$ women $(P<0.05)$ suggests that pregnant women are twice at odds for developing VVC as compared to non-pregnant women. The higher positivity in patients of vaginal discharge with pruritus was found to be statistically significant $(P<0.05)$. One in three patients was found to be positive for VVC and NAC was more prevalent as compared to candida albicans. This study concluded that pregnant women are at risk for VVC.
\end{abstract}

Keywords: Vulvo-vaginal candidiasis, C. albicans, non-albicans Candida (NAC), Pregnancy.

\section{INTRODUCTION}

Vulvo-vaginal Candidiasis (VVC) is defined as a condition with signs and symptoms of inflammation in the presence of Candida species in spite of other etiology (1). Candida is believed to be one of the most common causes of vaginal infections accounting for an estimated $17 \%$ to $39 \%$ cases (2). Approximately $75 \%$ of women experience at least one episode of VVC during their life (3). However, an estimated 5\% of women with vulvo-vaginal candidiasis experience recurrent vulvo-vaginitis (RVV), which is defined as four or more distinct episodes of vaginitis in a single year $(4,5)$.

Women having VVC present with spectrum of manifestations ranging from asymptomatic colonization to severe acute symptomatic infection (6). Certain patients may develop primarily vulvar symptoms instead of vaginal manifestations of VVC. Vulval pruritus and burning are the main symptoms and these are frequently accompanied by soreness, irritation, dyspareunia and dysuria. Vulval and vaginal erythema, edema, fissures and thick vaginal discharge are other common symptoms (7).

Prevalence of VVC increases in certain groups, such as pregnant or diabetic women, those using oral contraceptives and after antibiotic treatment (8). The incidence of candidiasis is almost doubled in pregnant women particularly in the third trimester compared to the non-pregnant women (9). VVC in the expectant women can cause abortion, chorio-amnionitis and subsequent preterm delivery. Premature neonates are severely endangered by generalized fungal infection because of their immature immune system (10).

VVC is the most common fungal infection in the world and any information on its distribution and etiology is useful for epidemiological purpose. Data related to this context is very limited in our region.

\section{MATERIALS AND METHODS}

Sampling criteria. This observational cross-sectional study was carried out in the Department of Microbiology, at a tertiary care hospital of central India between January 2016 and June 2017. All female patients in the reproductive age group (15-49 years) fulfilling the clinical criteria of vaginitis i.e. those with vaginal discharge, irritation, itching with or without pain and willing to give consent for the study were invited to participate. Vaginal swabs showing evidence of bacterial and protozoal infection and patients not willing to give consent were excluded. Consecutive sampling was followed to reach the desired sample size of 168

Specimen and tests. Three high vaginal swabs were collected from each patient using sterile cotton-tipped swabs. Collected specimen was transported immediately (within 30 minutes) to the laboratory in sterile tubes without using transport media. One swab was used for wet mount and second one for Gram staining (11). Third swab was used for culture on Sabouraud's Dextrose agar (SDA). Inoculated SDA culture tubes were incubated at $25^{\circ} \mathrm{C}$ for three to four days. Identification of culture growth was by colony characteristics (smooth, white to cream colored colonies were identified as those of candida); Gram's staining (round to oval Gram positive budding cells with or without pseudo-hyphae were considered as positive for candida). Germ tube test was carried out as a confirmatory test for Candida spp. For 
species identification various tests were used including Chlamydospore formation, sugar fermentation test, growth on CHROM agar (BD Diagnostic Systems Europe, Heidelberg, Germany) as described in previous studies (12, 13).

Statistical analysis. Data was analysed using SPSS version 20. Descriptive statistics in form of frequencies and percentages were used to illustrate the basic and disease characteristics of the study population. For quantitative analysis, univariate analysis using chi-square test and Fisher's exact test was used. The odds-ratio (OR) associated with each potential risk factor and the 95\% confidence interval (CI) were calculated. All results were considered significant at $P$ values less than 0.05 .

\section{RESULTS AND DISCUSSION}

A total of 168 subjects were included in the study. Of these, $76.2 \%$ subjects were non-pregnant and $23.8 \%$ were pregnant (Table 1). Among the 168 subjects, vaginal swab of $55(32.7 \%)$ subjects showed pure growth of Candida species (Figure 1). Table 2 represents distribution of Candida species. The nonalbicans Candida (NAC) species were predominant with $65.4 \%$. The most common species isolated on the basis of colony characteristics, morphology on corn meal agar, germ tube and chlamydospore formation, fermentation and assimilation reactions was $C$. albicans $(34.6 \%)$ followed by $C$. tropicalis $(23.6 \%)$ and C. glabrata $(21.8 \%)$.

Table 1. Basic characteristics of the study subjects

\begin{tabular}{llcc}
\hline \multicolumn{1}{c}{ Variable } & Frequency & Percentage \\
\hline Age & Upto 20 & 8 & 4.76 \\
& $21-30$ & 82 & 48.81 \\
& $31-40$ & 61 & 36.31 \\
Pregnancy & More than 40 & 17 & 10.12 \\
status & Pregnant & 40 & 23.80 \\
Risk factors & Non-Pregnant & 128 & 76.20 \\
& None & 59 & 35.12 \\
& Diabetes & 3 & 1.78 \\
& Gravida>1 & 86 & 51.20 \\
& BOH & 13 & 7.73 \\
& OCP use & 5 & 2.97 \\
& BOH+DM & 2 & 1.2 \\
\hline
\end{tabular}

$\mathrm{BOH}=\mathrm{Bad}$ obstetric history; $\mathrm{DM}=$ Diabetes mellitus; $\mathrm{OCP}=$ Oral contraceptive pill.

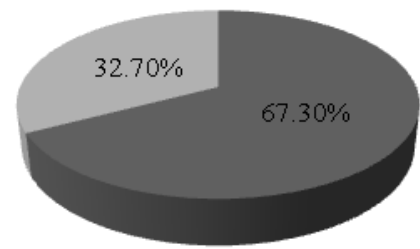

- Candida negative Candida positive

Table 2. Distribution of Candida species isolated from study participants $(n=55)$

\begin{tabular}{cc}
\hline Candida species & $\mathbf{n}(\boldsymbol{\%})$ \\
\hline C.albicans & $19(34.6)$ \\
Non albicans & $36(65.4)$ \\
C. dublinensis & $04(7.3)$ \\
C. glabrata & $12(21.8)$ \\
C. guilliermondi & $01(1.8)$ \\
C. krusei & $01(1.8)$ \\
C. lusitaniae & $03(5.5)$ \\
C. parapsilosis & $02(3.6)$ \\
C. tropicalis & $13(23.6)$ \\
\hline
\end{tabular}

Table 3 shows the Candida positivity across different age groups. Maximum Candida positivity was seen in age group 21-30 years (60\%) followed by age group 31-40 years $(32.72 \%)$. The higher positivity in age group 21-30 years was statistically significant in comparison to other age groups $(P<0.05)$. Table 4 presents information on the effect of risk factors on Candida positivity. Isolation of Candida was higher in women with one or more risk factors (94.5\%) as compared to those without any risk factors. Candida positivity was found to be higher among pregnant $(45.0 \%)$ as compared to non-pregnant (28.9\%) subjects included in the study. This difference in positivity among pregnant and non-pregnant women was not statistically significant $(P>0.05)$. The odds ratio suggests that pregnant women are twice at odds for developing VVC as compared to non-pregnant women. Candida positivity in patients having history of diabetes mellitus together with bad obstetrical history was $100 \%$. This was followed by that in patients presenting with $\mathrm{BOH}$ alone $(61.5 \%)$, pregnancy $(45 \%)$, diabetes mellitus alone (33.3\%), multigravida $(25.6 \%)$ and those taking oral contraceptives (20\%). However, none of these differences were statistically significant.

Table 5 shows signs and symptoms in study participants. Vaginal swab of 54 ( $96.4 \%$ ) out of 56 subjects presenting with thick curdy white discharge is the characteristic of vaginal candidiasis that were positive for Candida, in contrast to only $1(0.9 \%)$ of 112 subjects presenting with thin white non-curdy discharge and this difference was found to be significant statistically $(P<0.05)$. Isolation of Candida was the highest in women presenting vaginal discharge with pruritus $(56.9 \%)$, followed by those with dyspareunia or pruritus, dysuria and discharge $(33.3 \%$ each). Dysuria with vaginal discharge

Table 3. Distribution of Candida by age

\begin{tabular}{cccc}
\hline & & & \\
Up to 20 & $08(4.76)$ & $02(3.64)$ & 1.20 \\
$\mathbf{2 1 - 3 0}$ & $82(48.81)$ & $33(60.0)$ & 19.64 \\
$\mathbf{3 1 - 4 0}$ & $61(36.31)$ & $18(32.72)$ & 10.71 \\
More than 40 & $17(10.12)$ & $02(3.64)$ & 1.20 \\
Total & 168 & 55 & 32.75 \\
\hline
\end{tabular}

accounted for $25 \%$ positivity and vaginal discharge alone for $20.2 \%$ Candida positivity. The higher positivity in patients of vaginal discharge with pruritus was found to be statistically significant $(P<$ $0.05)$. Thick curdy white discharge was found to be associated with confirmed vaginal candidiasis in $96.3 \%$ of such subjects. Additional presence of either excoriation or vulval swelling further strengthened the diagnosis, as $100 \%$ of such subjects grew Candida spp. 
Table 4. Effect of risk factors on Candida positivity

\begin{tabular}{|c|c|c|c|c|c|}
\hline Risk factors & & Candida positive n (\%) & Candida negative & OR and CI $95 \%$ & $P$ value \\
\hline Risk factor Present & 149 & $52(34.9)$ & $97(65.1)$ & 2.8591 & 0.1072 \\
\hline Risk factor Absent & 19 & $3(15.8)$ & $16(84.2)$ & [0.7963 to 10.2660$]$ & \\
\hline \multicolumn{6}{|c|}{ Type of risk factor } \\
\hline \multicolumn{6}{|l|}{ Pregnancy } \\
\hline Yes & 40 & $18(45.0)$ & $22(55)$ & 2.0123 & \\
\hline No & 128 & $37(28.0)$ & $91(72)$ & [0.9690 to 4.1790$]$ & 0.0607 \\
\hline Gravida $>1$ & & & & 0.8818 & \\
\hline Yes & 86 & $22(25.6)$ & $64(74.4)$ & [0.4453 to 1.7462$]$ & 0.7182 \\
\hline No & 82 & $23(28.1)$ & 59 (71.9) & & \\
\hline \multicolumn{6}{|l|}{$\mathrm{BOH}$} \\
\hline Yes & 13 & $8(61.5)$ & $5(38.5)$ & $* *$ & \\
\hline No & 155 & $47(30.4)$ & $108(69.6)$ & & \\
\hline \multicolumn{6}{|l|}{ DM } \\
\hline Yes & 3 & $1(33.3)$ & $2(66.6)$ & $* *$ & \\
\hline No & 165 & $54(32.7)$ & $111(67.2)$ & & \\
\hline \multicolumn{6}{|l|}{$\mathrm{OCP}$} \\
\hline Yes & 5 & $1(20.0)$ & $4(80)$ & $* *$ & \\
\hline No & 163 & $54(33.2)$ & $109(66.8)$ & & \\
\hline \multicolumn{6}{|l|}{$\mathrm{BOH}+\mathrm{DM}$} \\
\hline Yes & 2 & $2(100)$ & $0(0)$ & $* *$ & \\
\hline No & 166 & $53(32.0)$ & $113(68.0)$ & & \\
\hline
\end{tabular}

$\mathrm{BOH}=\mathrm{Bad}$ obstetric history DM = Diabetes mellitus; $\mathrm{OCP}=$ Oral contraceptive pill

** Fisher exact test was used

Table 5. Signs and symptoms in study participants

\begin{tabular}{lcc}
\hline Symptoms & $\begin{array}{c}\text { No. of subjects, } \\
\mathbf{n = 1 6 8}(\mathbf{\%})\end{array}$ & $\begin{array}{c}\text { Candida positive, } \\
\mathbf{n = 5 5}(\mathbf{\%})\end{array}$ \\
\hline Vaginal discharge alone & $94(55.95)$ & $19(34.55)$ \\
Vaginal discharge + Pruritus & $51(30.36)$ & $29(52.72)$ \\
Vaginal discharge + Dysuria & $08(4.76)$ & $02(3.64)$ \\
Vaginal discharge + Dyspareunia & $09(5.36)$ & $03(5.45)$ \\
Vaginal discharge + Pruritus + Dysuria & $06(3.57)$ & $02(3.64)$ \\
Signs & & $52(94.5)$ \\
Thick curdy white discharge alone & $54(32.14)$ & $1(1.81)$ \\
Thin non-curdy white discharge alone & $109(64.88)$ & $1(1.81)$ \\
Thick curdy white discharge + Excoriation & $01(0.59)$ & $0(0)$ \\
Thin non-curdy white discharge + Excoriation & $02(1.19)$ & $1(1.81)$ \\
Thick curdy white discharge + Vulval swelling & $01(0.59)$ & $0(0)$ \\
Thin non-curdy white discharge + Vulval swelling + Excoriation & $01(0.59)$ & \\
\hline
\end{tabular}

In the present study, VVC was found in about one in three of the symptomatic women. This is in concordance with two reports from India that is $31.1 \%$, and $37 \%$ on a similar study group $(14,15)$. Onifade et al. (16) from Nigeria $(81.5 \%)$ reported a very high prevalence. These differences may be attributed to several factors including the type of patient studied, sample size, source of clinical specimens and presence of risk factors.

Recently, NAC species have been recovered with increasing frequency. In our study, the rate of isolation of NAC was higher than that of $C$. albicans. However, $C$. albicans was the most dominant species isolated. Higher isolation of NAC over $C$. albicans has also been reported by Kikani et al. (55.6\% vs. $44.4 \%$ ) (17), Babin et al. (64.5\% vs. 35.5\%) (18) and Namrata et al. (53\% vs. 47\%) (19). Earlier reports from Middle East region revealed higher rate of isolation of $C$. albicans. Cultural, ethnic and epidemiological differences may influence the isolation rate of different yeast from vulvo-vaginitis samples (20-23). However, it is critically important to isolate and identify Candida species for diagnosis, treatment and epidemiological purposes.

The age group, 21-30 years is the most sexually active age group. There is high risk of pregnancy, indiscriminate use of drugs, use of contraceptive pills and other protective family planning devices to prevent pregnancy. AIDS and other sexually transmitted diseases are frequent in this age group. All these factors promote VVC and can be a reason for higher prevalence of VVC in this particular age group. Another physiological reason is that during this period, the ovary produces high amount of estrogen, which favors the growth of Candida by maintaining the acidic $\mathrm{pH}$ and enhancing the yeast adherence to vaginal epithelial cells. In the present study, incidence of VVC in age group 21-30 years was 40.2\%. The higher incidence of VVC in the age group of 20-30 years was also reported in previous reports (24-28). A similar case study in Libya, demonstrated the highest positivity in age group $15-25$ years $(44.4 \%)$ which 
was found to be associated with early age of marriage (29).

Vaginal discharge alone was reported to be the predominant sign/symptom observed in cases of VVC by other workers (30-34).In this study we found $20.2 \%$ cases with thick cheesy vaginal discharge which is similar to previous reports $(30,31)$. Maximum Candida positivity in the present study was seen in patients with thick curdy white discharge $(96.4 \%)$ as compared to thin non-curdy white discharge $(0.9 \%)$ and this difference was statistically significant $(P<0.05)$. Fule et al. $(15)$ found this to be the major symptom in $52.4 \%$ of their subjects of VVC and Ugwa et. al. (32) found this to be the most common symptom in $47.4 \%$ of their cases. Candida positivity in patients presenting vaginal discharge plus one or more of the other symptoms was $48.7 \%$ in this study which is similar to another report $(47.5 \%)$ by Fule et al. (15).

Vaginal discharge with pruritus $(56.9 \%)$ was a major symptom in Candida positive cases in the present study $(P<0.05)$. Similar result was reported by other researchers and demonstrated in $63.2 \%$ cases $(31)$. Dysuria with pruritus was seen in $33.3 \%$ cases and dysuria alone in $25 \%$ Candida positive cases was observed in the present study. Vaginal discharge with dysuria was also observed in $18 \%$ (10) and $12 \%$ (33) cases in other studies which is less than our findings. One third of our cases presenting with dyspareunia were positive for Candida which is higher than another previous report (33).

On analyzing the predisposing risk factors, in the present study we observed that VVC was confirmed in $45 \%$ of pregnant women whereas in non-pregnant women it was confirmed in $28.9 \%$ cases and this difference was not statistically significant. Several previous studies reported a similar finding of higher prevalence in pregnant as compared to non-pregnant $(24,29,30,35)$. Another study also reported pregnancy to be the most typical predisposing risk factor $(29.75 \%)$ for VVC (18) and also, been cited by other researchers $(33,36)$. Increased level of vaginal glycogen, high level of reproductive hormones and immune-suppression during pregnancy has been attributed as important risk factors for VVC. Emotional stress during pregnancy has also been incriminated as a major risk factor for VVC.

Several studies $(19,37,38)$ found oral contraceptive pill OCP to be a risk factor for VVC. Our observation of association of oral contraceptive as predisposing factor was seen in $20 \%$ cases. Estrogen facilitates the adherence of yeast to the vaginal epithelium; hence high estrogen in oral contraceptives may be one of the contributing factors.

In the present study, $33.3 \%$ of women with diabetes mellitus alone had confirmed VVC and $100 \%$ of those who had diabetes mellitus along with bad obstetrics history confirmed VVC. These results show resonance with previous studies (39-41). Earlier studies on diabetic women in developed countries demonstrated wide variations in prevalence rates starting from $7 \%$ to $>50 \%(42-44)$. In diabetic patients, especially among type I diabetes, hyperglycemia limits neutrophil function. The neutrophils are unable to phagocytose and kill Candida and thus there is a higher incidence of candidiasis in diabetes patients. Diabetes is a proven predisposing factor for vulvovaginal infection especially for vaginal candidiasis, along with pregnancy, use of broad-spectrum antibiotics, high-estrogen-dose, oral contraceptives, obesity and drug addiction (45).

In the present study, $25.6 \%$ of multigravidas were positive for VVC. Other studies have reported a significantly higher prevalence in multigravidas as compared to primigravida women $(33,46)$.

The limitation of our study was its small sample size which was collected from just one tertiary care hospital in central India. Due to this constraint, the results of this study cannot be generalized. However, this study provides important information about the pattern and risk factors of VVC infection in women of reproductive age in central India. It is recommended to take up further study of $C$. albicans and NAC as important pathogens, especially in high risk groups.

\section{REFERENCES}

1. Jacqueline MA and Bettina CF. 2010. Candida Infections of the Genitourinary Tract. Clin. Microbiol. Rev. 23:253-273.

2. Anderson MR, Klink K and Cohrssen A. 2004. Evaluation of vaginal complaints. JAMA. 291:1368-79.

3. Sobel JD, Geiger AM and Foxman B. 1995. Chronic Vulvo-vaginal candidosis: characteristics of women with Candida albicans, $C$ glabrata and no Candida. Genitourinary Med. 71:304-307.

4. Güzel AB, Aydın M, Meral M, Kalkancı A and Ilkit M. 2013. Clinical characteristics of Turkish women with Candida krusei vaginitis and antifungal susceptibility of the C. krusei isolates. Infect. Dis. Obstet. Gynecol. 2013:698736.

5. Zhou X, Westman R, Hickey R, Hansmann MA, Kennedy C, Osborn TW et al. 2009.Vaginal microbiota of women with frequent vulvovaginal candidiasis. Infect. Immune. 77:4130-4135.

6. Sobel JD, Faro S, Frocs RW, Foxman B, Ledger WJ and Nyirjesy PR. 1998. Vulvo-vaginal candidiasis: Epidemiological, diagnostic and therapeutic considerations. Am. J. Obstet. Gynecol. 178:203-11.

7. Nyirjesy P. 2008. Vulvo-vaginal candidiasis and bacterial vaginosis Infect. Dis. Clin. North. Am. 22:637-52.

8. Kumari V, Banerjee T, Kumar P, Pandey S and Tilak R. 2013 Emergence of non-albicans Candida among candida vulvovaginitis cases and study of their potential virulence factors from a tertiary care center, North India. Indian J. Path. Microbiol. 56:144-147.

9. Alo MN, Anyim C, Oyebuchi AK and Okonkwo EC. 2012. Prevalence of asymptomatic co-infection of Candidiasis and Vaginal Trichomoniasis among Pregnant Women in Abakaliki South Eastern Nigeria. Nat. Sci. Res. 2:87-91.

10. Kanagal DV, Vineeth VK, Kundapur R, Shetty H and Rajesh A. 2014 Prevalence of Vaginal Candidiasis in Pregnancy among Coastal South Indian Women. J. Womens Health. Issues Care 3:6.

11. Chander J. 2011. Candidiasis. Textbook of Medical Mycology; $3^{\text {rd }}$ edition. 275:507-530.

12. Chakrabarti A et al. 2008. Medical Mycology Laboratory Procedures PGIMER, Chandigarh. 57-68.

13. Nadeem SG, Hakim ST and Kazmi SU. 2010 Use of CHROM aga Candida for the presumptive identification of Candida species directly from clinical specimens in resource limited settings. Libyan J. Med. $9 \cdot 5$

14. Jombo GTA, Opajobi SO, Egah DZ, Banwat EB and Akaa PD. 2010 Symptomatic vulvo-vaginal candidiasis and genital colonization by Candida species in Nigeria. J. Public Health Epidemiol. 2:147-51.

15. Fule SR, Das D and Fule RP. 2015. Detection of phospholipase activity of Candida albicans and non-albicans isolated from women of reproductive age with vulvo-vaginal candidiasis in rural area. Indian J. Med. Microbiol. 33:92-95.

16. Onifade AK and Olorunfemi OB. 2005. Epidemiology of vulvo-vaginal candidiasis in female patients in Ondo State Government Hospital. J. Food Agric. Environ. 3:118-9. 
17. Kikani B, Kikani K and Pathak S. 2008. Effects of chemically synthesized azole compounds on clinical isolates of vaginal candidiasis, in comparison with commercially available antifungal drugs. Internet J. Microbiol. 4:1-7.

18. Babin DK, Rao SP and Rao TV. 2013. Clinico-mycological profile of vaginal candidiasis in a tertiary care hospital in Kerala. Int. J. Res. Bio. Sci. 3:55-59.

19. Kalia N, Singh J, Sharma S, Kamoj S, Arora H and Kaur M. 2015. Prevalence of Vulvo-vaginal Infections and species specific distribution of vulvo-vaginal candidiasis in married women of north India. Int. J. Cur. Microbiol. Appl. Sci. 4:253-266.

20. El-sayed H and Hamouda A. 2007. Candida albicans causing vulvovaginitis and their clinical response to antifungal therapy. Egypt J. Med. Microbiol. 16:53-62.

21. Alfouzan W, Dhar R, Ashkanani H, Gupta M, Rachel C and Khan ZU. 2015. Species spectrum and antifungal susceptibility profile of vaginal isolates of Candida in Kuwait. J. Mycol. Med. 25:23-8.

22. Al-mamari A, Al-buryhi M, Al-heggami MA and Al-hag S. 2014 Identify and sensitivity to antifungal drugs of Candida species causing vaginitis isolated from vulvo-vaginal infected patients in Sana'a city. Der. Pharma. Chemica. 6:336-42.

23. Al-Hedaithy S. 2002. Spectrum and proteinase production of yeasts causing vaginitis in Saudi Arabian women. Med. Sci. Monit. 8:498-501.

24. Nwadioha SI, Egah DZ, Alao OO and Iheanacho E. 2010. Risk factors for vaginal candidiasis among women attending primary health care centers of Jos, Nigeria. J. Clin. Med. Res. 2:110-3.

25. Bankar SM, Powar RM, Patil SA and Kalthur SG. 2012. Prevalence of non-albicans Candida infection in Maharashtrian women with leucorrhea. Ann. Trop. Med. Public Health 5:119-23.

26. Sehgal SC. 1990. Epidemiology of male urethritis in Nigeria. J. Trop. Med. Hyg. 93:151-152.

27. Tamsikar J, Singh SM and Naidu J. 2011. Prevalence of non-Candida albicans vaginitis in patients on hormonal treatment in Jabalpur. Central India. J. Med. Mycol. 1:256-9.

28. Samuel O, Ifeanyi O and Ugochukwu O. 2015. Prevalence of Candida Species among Vaginitis Symptomatic Pregnant Women Attending Ante-natal Clinic of Anambra State University Teaching Hospital, Awka, Nigeria. Bioengg. Biosci. 3:23-27.

29. Altayyar IA, Alsanosil A and Osman NA. 2016. Prevalence of vaginal candidiasis among pregnant women attending different gynecological clinic at South Libya. Europ. J. Exper. Biol. 6:25-29.

30. Dias LB, de Souza C, Melhem M, Szeszs MW, Filho JM and Hahn RC. 2011. Vulvo-vaginal candidiasis in Mato Grosso, Brazil: pregnancy status, causative species and drugs tests. Braz. J. Microbiol. 42:1300-7.

31. Narayankhedkar A, Hodiwala A and Mane A. 2015. Clinicoetiological Characterization of Infectious Vaginitis amongst Women of
Reproductive Age Group from Navi Mumbai, India. J. Sex. Transm. Dis. 2015:817092

32. Ugwa EA. 2015. Vulvo-vaginal Candidiasis in Aminu Kano Teaching Hospital, North-West Nigeria: Hospital-Based Epidemiological Study. Ann. Med. Health Sci. Res. 5:274-278.

33. Aslam M, Hafeez R, Ijaz S and Tahir M. 2008. Vulvo-vaginal candidiasis in pregnancy. Biomedica. 24:54-6.

34. Altayyar IA, Alsanosi AS and Osman NZ. 2016. Prevalence of vaginal candidiasis among pregnant women attending different gynecologica clinic at South Libya. Europ. J. Exper. Biol. 6:25-29.

35. Jindal N, Agrawal A and Gill P. 2006. Significance of Candida culture in women with vulvo-vaginal symptoms. J. Obstet. Gynecol. India 56:139-41

36. Okungbowa F, Isuehuemhen O and Dede A. 2003. The Dis- tribution, Frequency of Candida Species in the Genitou-rinary Tract among Symptomatic individuals in Nigeria cities, Revised Iberoam Microbiol 20:60-63

37. Dressen G, Kusche W, Neumeister C and Schwantes U. 2012 Diagnosis of Vulvo-vaginal Candidiasis and Effectiveness of Combined Topical Treatment with Nystatin: Results of a Non-Interventional Study in 973 Patients. The Open Women's Health J. 6:19-23.

38. Linhares IM, Witkin SS, Miranda SD, Fonseca AM, Pinotti JA and Ledger WJ. 2001. Differentiation between women with vulvo-vaginal symptoms who are positive or negative for Candida species by culture. Infect. Dis. Obstet. Gynecol. 9:221-5.

39. Goswami D, Goswami R and Banerjee U. 2006. Pattern of Candida species isolated from patients with diabetes mellitus and vulvo-vagina candidiasis and their response to single dose oral fluconazole therapy. J Infect. 52:111-7.

40. Peer AK, Hoosen AA, seedat MA, van den Ende J and Omar MA 1993. Vaginal yeast infections in diabetic women. Afr. J. Med. 83:9 727.

41. Vivek KS, Deepali S, Archana S and Asha MJ. 2015. Prevalence of vaginal candidiasis in diabetic women of Madhya Pradesh, India. Int. J. Curr. Microbiol. App. Sci. 4:834-846.

42. Bohannon NJV. 1998 Treatment of Vulvo vaginal Candidiasis in Patients with Diabetes. Diabetes care 21:451-6.

43. Davis C. 1986. CMI description sheets. Mycopathologia. 96:171-3.

44. Malazy OT, Shariati M, Heshmat R, Majlesi F, Alimohammadian M and Moreira D. 2006. Vulvo vaginal candidiasis. Inter. J. Obstet. 92:266-267.

45. Rajawat AS, Shrivastava V, Shrivastava A and Singh V. 2013. In vitro evaluation of inhibitory activity of Probiotic Lactobacilli against Candidaspecies isolated from the vaginal flora of Immunocompromised Patients. South Asian J. Exp. Biol. 3:325-329.

46. Omar AA. 2001. Grams stain Vs Culture in the diagnosis of VVC. East Mediter. Health J. 7:925-934. 